\title{
Les voix du "moi" dans Enfance de Nathalie Sarraute
}

\section{Voices of the self in Nathalie Sarraute's Enfance}

\author{
ANTONELLA LIPSCOMB \\ Investigadora independiente \\ antonella_lipscomb@hotmail.com
}

\begin{abstract}
If autobiography is the privileged field of the first person narrative, Nathalie Sarraute adopts in Enfance (1983) an original procedure: the dialogue. In her autobiography, the alternation between first and second person singular enable to simulate a dialogue between two instances of the self. This article entitled "Voices of the self in Nathalie Sarraute's Enfance" aims to analyse this interior duality orchestrated by the two narrative voices where the implicit voices that have shaped the writer's childhood - voices of the mother, the father and the stepmother Véra - emerge from the past. A polyphony of living voices, as intact as the memories evoked.
\end{abstract}

Key-words

Nathalie Sarraute, autobiography, dialogue, voices.

\section{Resumen}

Si la autobiografía constituye el reino privilegiado del relato en primera persona, Nathalie Sarraute adopta en Enfance (1983) un procedimiento original: el diálogo. En su relato, la alternancia de la primera y la segunda persona del singular permite simular un diálogo entre dos instancias de un mismo "yo". El presente artículo analizará este desdoblamiento interior orquestado por dos voces narrativas donde afloran las voces implícitas que han marcado el pasado de la autora - las voces de la madre, del padre y de la madrastra, Véra. Toda una polifonía de voces vivas, tan intactas como los recuerdos evocados.

\section{Palabras clave}

Nathalie Sarraute, autobiografía, diálogo, voces. 


\section{Introduction}

Au cours des années 80, les nouveaux romanciers Alain Robbe-Grillet et Nathalie Sarraute se tournent vers le genre autobiographique, suscitant aussi bien l'étonnement du public que celui de la critique. Comme l'explique Armel, ces initiateurs de la révolution romanesque des années 1950 "sortent de leur réputation de purs théoriciens de l'écriture, soi-disant irréductibles à tout discours sur eux-mêmes, en livrant au public des livres à la première personne, qui ne s'intitulent plus ni 'roman' ni 'récit', ni encore moins 'essai', où le narrateur-personnage principal est clairement identifié à l'auteur" (Armel, 1990: 25). "Il ne s'agit pas d'une autobiographie. Ce n'est pas un rapport sur toute ma vie" (Forrester, 1983: 19), se défend Sarraute au sujet de Enfance (1983). Ailleurs elle affirme également:

Je n'aime pas l'autobiographie, je n'ai aucune confiance dans les autobiographies, parce qu'on s'y décrit toujours sous un jour..., on veut se montrer sous un certain jour. Et puis c'est toujours très partial; enfin, moi, je n'y crois jamais ${ }^{1}$.

Mais plus loin, Sarraute rectifie: “Ecoutez, je crois qu'un des textes que j'admire le plus de tous les textes littéraires, ce sont Les Confessions de Rousseau. Alors, ça suffirait déjà pour que je ne puisse pas dire du mal d'une autobiographie"2. Si l'autobiographie est le domaine privilégié du récit à la première personne, Sarraute adopte dans Enfance un procédé original: le dialogue. Un procédé que Simone de Beauvoir, dans Mémoires d'une jeune fille rangée, se rappelle également avoir adopté le jour de ses dix-neuf ans, lorsque dans la bibliothèque de la Sorbonne elle s'était mise à écrire "un long dialogue où alternaient deux voix qui étaient toutes deux les [s]iennes: l'une disait la vanité de toutes choses, et le dégoût et la fatigue; l'autre affirmait qu'il est beau d'exister, fût-ce stérilement" (de Beauvoir, 1958: 320). Le dialogue autobiographique n'est donc pas un procédé nouveau, il avait d'ailleurs déjà été utilisé par Jean-Jacques Rousseau dans ses Dialogues, surtitrés Rousseau juge de Jean-Jacques. Dans ce dialogue fictif, un personnage nommé "Rousseau", qui a lu les écrits de l'écrivain "Jean-Jacques" mais ne le connaît pas, débat avec un autre personnage nommé “Le Français" qui lui connaît “Jean-Jacques” mais n'a pas lu ses écrits. L’auteur de ces Dialogues y reconstitue donc fictivement un procès où l'accusateur, "Le Français", et le défenseur, "Rousseau", se débattent autour d'une même cause: “Jean-Jacques", auteur et inventeur du procès. Le but de l'ouvrage est d'examiner le pour et le contre de l'homme public: "Jean-Jacques". Une démultiplication du sujet en trois instances fictives qui, comme le constate Lecarme, "se fonde sur la certitude de Rousseau que ses Confessions [...] ont échoué et échoueront à le réconcilier avec le genre humain" et ouvre une nouvelle voie vers la recherche de soi (Lecarme \& Lecarme-Tabone, 1997: 152). C'est cette voie qu'entreprend

1 Emission du 5 avril 1984 de Jean Montalbetti, sur France-Culture: Entretien avec Nathalie Sarraute, cité in Jacques Lecarme \& Eliane Lecarme-Tabone (1997: 15).

2 Ibid. 
Sarraute dans Enfance où un autre dialogue fictif entre deux voix explore les profondeurs de son "moi". Un dédoublement intérieur orchestré par deux voix narratives d'où émergent les voix implicites qui ont marqué le passé de l'auteur - la voix de la mère, du père et de la bellemère Véra. Toute une polyphonie de voix vivantes, aussi intactes que les souvenirs évoqués.

\section{Voix narratives}

Dans ses Essais sur le roman Michel Butor explique que pour qu'il y ait dialogue il faut "que le personnage en question, pour une raison ou pour une autre, ne puisse pas raconter sa propre histoire, que le langage lui soit interdit, et que l'on force cette interdiction" (Butor, 1992: 80). En effet, continue-t-il, "si le personnage connaissait entièrement sa propre histoire, s'il n'avait pas d'objections à la raconter ou se la raconter, la première personne s'imposerait: il donnerait son témoignage" (Butor, 1992: 81). Si le dialogue s'instaure par conséquent dans Enfance, c'est que la narratrice a besoin d'être secondée par une deuxième voix pour relier les éléments nécessaires à la reconstitution d'un véritable témoignage, et récupérer certains instants du passé. Grâce au dialogue le lecteur assisterait donc à la révélation d'un passé enfoui, caché, et à la naissance d'un langage capable de l'évoquer. Plus que "personnage", terme que Sarraute rejette en ce qu'il désigne quelque chose de rigide et de fixé, il s'agit dans Enfance de voix: une voix féminine qui prend en charge la narration, et une voix qui porte les marques du masculin et s'occupe le plus souvent à poser des questions. Si le "je" explicite de la première voix se confond avec le "je" de l'auteur, le "je" implicite de la deuxième voix est plus difficile à identifier. C'est la marque du masculin, présente dans les adjectifs "grandiloquent" et "outrecuidant", qui porte le lecteur à confusion (Sarraute, 1983: 8-9). Mais peutêtre est-ce justement lui, le lecteur, que l'auteur aurait voulu suggérer à travers cette deuxième voix. C'est d'ailleurs ainsi que Sarraute compare cette instance qui l'aide, d'après elle, à "remettre les choses en place [...] un côté qui relit et demande: "pourquoi dis-tu cela?"”; en d'autres mots, "un double, ce lecteur idéal que tout écrivain projette", déclare-t-elle en effet (Gosselin, 1996: 31). Difficile cependant, comme le suggère Yvette Went-Daoust, de parler de "collaboration fonctionnelle" entre lecteur et auteur lorsque Sarraute "connaît par avance toutes les questions et réponses" (Went-Daoust, 1987: 344). Aussi vaudrait-il mieux, comme le propose Monique Gosselin, interpréter cette voix comme celle d'un "double où s'amalgament une instance du moi et une figure intériorisée du lecteur fidèle " (Gosselin, 1996: 31).

C'est par une question très directe de ce "double", cette deuxième voix, que Sarraute choisit d'ouvrir son récit: "-Alors, tu vas vraiment faire ça? Evoquer tes souvenirs d'enfance" (Sarraute, 1983: 7). Difficile de ne pas ressentir la gêne qui entoure cette expression; elle est si sujette au cliché que sa seule énonciation conduit l'auteur à "tortiller" (Sarraute, 1983: 7). Or, c'est bien cette formule que Sarraute choisit pour définir son entreprise: “Oui, je n’y 
peux rien, ça me tente, je ne sais pas pourquoi..." (Sarraute, 1983: 7). Si l'enfance est pour de nombreux écrivains une tentation dangereuse, la mémoire n'offrant rien de plus incertain, elle constitue néanmoins un détour obligé pour l'auteur âgé. C'est ce que suggèrent François Mittérand et Elie Wiesel dans cet autre dialogue à deux voix, mais réel cette fois, des $M e ́-$ moires à deux voix:

E. W.: - Avec l'âge, vient le moment où l'on se met à penser avec insistance, à sa propre enfance.

F. M.: - Oui. C'est peut-être un phénomène constant, inévitable, lorsqu'on vieillit et que l'on approche de la mort. On revient à ses origines, à ses premières émotions, à ses premières sensations (Wiesel \& Mittérand, 1995: 26).

C'est un même désir de retrouver certaines "émotions" et "sensations" qui ont marqué son enfance que partage Sarraute. Mais c'est surtout pour continuer à chercher ce qui l'a toujours intéressée, à savoir ces mouvements intérieurs qu'elle nomme les “tropismes", qu'elle se laisserait tenter. Si Sarraute place par conséquent l'expression "souvenirs d'enfance" entre guillemets c'est qu'elle craint que son récit sacrifie ce "mouvement" qui est encore "tout vacillant", "des petits bouts de quelque chose d'encore vivant", pour quelque chose de "fixé une fois pour toutes" (Sarraute, 1983: 9). Dans un entretien avec Serge Faucherau et Jean Ristat, Sarraute explique:

Je n'ai choisi dans Enfance que des souvenirs dans lesquels existaient ces mouvements, mais lorsque j'ai voulu faire revivre mes vacances en Isère, les images se sont animées [...] Je n'écrivais pas une autobiographie dans laquelle je devais raconter tout ce qui m'était arrivé. (in Gosselin, 1996: 25-26)

Ce ne sont donc pas tous les souvenirs de son enfance que l'auteur évoque ici, mais uniquement ceux qui l'ont marquée et qui continuent encore maintenant à la toucher. Le récit se construit en effet à partir du refus du "tout cuit" et de l'effort de saisir une substance pré-linguistique capable de traduire "ces quelques moments, quelques mouvements [...] encore intacts, assez forts pour se dégager de cette couche protectrice qui les conserve" (Sarraute, 1983: 227). Comme le constate Valérie Minogue, le lecteur n'est pas plongé dans l'enfance, mais dans le processus de remémoration et écriture de l'enfance, un processus considéré partiel, sélectif et interprétatif (Minogue, 1986: 73)³. Aussi le récit se termine-t-il lorsque survient cet "énorme espace très encombré, bien éclairé" de l'adolescence et de la vie adulte. Dans un autre entretien avec Simone Benmussa, Sarraute précise: "Je travaille à partir uniquement de ce que je ressens moi-même. Je ne me place pas à l'extérieur, je ne cherche pas à analyser du dehors" (Benmussa: 1987: 139-140). C'est donc bien à l'intérieur

3 "We are not plunged into a childhood, we are plunged into the process of remembering and writing it, a process acknowledged as partial, selective and interpretative". 
que se place Sarraute dans Enfance, où en devenant pour la première fois le sujet et objet de sa recherche fondamentale, elle expose son "moi" à travers ces deux voix.

L'objectif que Sarraute se propose d'atteindre dans cette autobiographie est représenté emblématiquement dans la première scène du récit. Séparée du dialogue qui ouvre Enfance par un simple blanc, cette scène se déroule à Interlaken, au début du siècle, dans le salon d'un grand hôtel. Armée d'une paire de ciseaux l'auteur, enfant, menace la soierie d'un canapé sous le regard réprobateur d'une gouvernante allemande:

'Ich werde es zerreissen'... 'Je vais le déchirer '... le mot 'zerreissen' rend un son sifflant, féroce dans une seconde quelque chose va se produire... je vais déchirer, saccager, détruire... (Sarraute, 1983: 11)

“Les paroles m'entourent, m'enserrent, me ligotent", explique encore la première voix, et soudain c'est "l'excitation, l'exaltation": les ciseaux ont percé la soie et "quelque chose de mou, de grisâtre s'échappe par la fente" (Sarraute, 1983: 12-13). Les mots prononcés alors par la première voix, "Voilà, je me libère", pourraient constituer une épigraphe appropriée pour l'œuvre entière: les ciseaux que l'enfant, Natacha, enfonce dans le canapé symbolisent en effet la transgression effectuée par l'auteur en évoquant son passé. Tout au long du dialogue, la première voix raconte et l'autre commente, un échange qui dote le récit d'un mouvement saccadé, balancé par un rythme qui avance, s'arrête, pour ensuite, semble-t-il, piétiner. C'est la première voix qui assume le récit et qui est chargée de faire resurgir son passé. $\mathrm{Si}$, par conséquent, elle hésite, se corrige, ou se tait, c'est qu'elle cherche, par approches successives, à cerner la sensation éprouvée. Une hésitation suggérée par les points de suspension, qui confèrent au récit son côté "vacillant", "vivant", et son impression d'être une recherche en "mouvement" (Sarraute, 1983: 131). La deuxième voix joue, en revanche, un rôle plus complexe: contrairement à la première voix qui se limite au rôle du souvenir émotif et factuel, elle passe son temps, d'après Lejeune, à osciller entre trois positions: "contrôle", "écoute" et “collaboration" (Lejeune, 1990: 33).

La première fonction de cette deuxième voix est "contrôle" et a pour arme le soupçon et pour objectif: "éviter de reconstruire et de romancer" (Lejeune, 1990: 33). Semblable à un censeur, cette seconde voix surveille la conformité des faits avec leur narration. Attentive à la moindre défaillance, elle se montre très rapide à détecter et à censurer toute déformation du passé ou souvenir lié au cliché. Un souci d'exactitude qui traduit, implicitement, l'effort d'authenticité et de fidélité entrepris par l'auteur quant à l'évocation de son passé. C'est, comme le remarque Bruno Vercier, l'interrogation et parfois plus radicalement la négation qui caractérisent cette première fonction:

L'interrogation en forme de dialogue permet à Sarraute de déjouer tous les pièges que pose l'écriture autobiographique, et en tout premier lieu l'empêche de succomber à la fascination de la nostalgie pour ce qu'elle appelle avec ironie les 'beaux souvenirs d'enfance'. (Vercier, 1987: 164) 
Même défiance pour les magnifiques "traumatismes de l'enfance" qui risquent de surgir à chaque fois qu'un souvenir douloureux est évoqué (Sarraute, 1983: 85). Au cours d'une maladie contagieuse contractée par l'enfant, la première voix se souvient de sa mère "assise à la table en train d'écrire" ou encore "dans un fauteuil en train de lire" (Sarraute, 1983: 37). Devinant le reproche implicite de la première voix devant l'apparente indifférence de la mère, la deuxième voix rectifie: “- Sois juste, il lui est arrivé pendant cette maladie de venir s'asseoir près de ton lit avec un livre" (Sarraute, 1983: 38). Plus loin, ce même sentiment d'abandon éprouvé par la première voix est encore une fois réprouvé par la deuxième:

Quand je lui dis que j'ai sommeil ou que je suis fatiguée, elle referme le livre très vite, il me semble qu'elle est contente de s'arrêter...

- Tu sentais cela vraiment à ce moment? (Sarraute, 1983: 39)

Ailleurs, la première voix évoque le souvenir des paroles "Véra est bête", lancées par sa mère la veille de leur séparation. Comparées à un "paquet [...] qu'on remet à son enfant qu'on va placer comme interne au collège" et auquel on rassure que ça pourra "servir" quand il sera "là-bas", ces paroles sont interprétées une seconde fois et moins dramatiquement par la deuxième voix:

- Non, là il faut que je t'arrête, tu te laisses entraîner [...] C'est toi qui l'as contrainte par tes questions [...] C'est parce qu'elle a fini par te céder qu'elle t'a répondu: 'L'oncle m'a dit que Véra est bête'. (Sarraute, 1983: 188)

Lorsque la première voix exagère encore au sujet du "chagrin" de son père tombé malade à la suite de la mort de sa fille, la deuxième voix corrige: “- C'est vrai qu'il avait énormément souffert de sa mort, mais il était tombé malade parce qu'il avait attrapé d'elle la scarlatine" (Sarraute, 1983: 118-119). Parfois la deuxième voix exerce sa fonction pour vérifier l'exactitude de certains faits narrés. Ainsi, la verra-t-on souvent demander des précisions: “- Est-il certain que cette image se trouve dans Max et Moritz? Ne vaudrait-il pas mieux le vérifier?" (Sarraute, 1983: 48). Ailleurs, au lieu de demander des précisions, la deuxième voix en apporte, comme lorsqu'elle s'applique à respecter la chronologie du souvenir évoqué:

- Je pense que c'est avant le séjour de grand-mère que cela devait se situer...

- Ou est-ce après ?

- Non, avant... il me semble que grand-mère est venue quand tu allais entrer dans la classe du certificat d'études... (Sarraute, 1983: 241)

La seconde voix non seulement exerce son contrôle sur le contenu de l'épisode raconté, mais elle veille également à la forme, à la façon dont l'épisode est évoqué. Dans l'exemple suivant, la première voix se souvient d'une scène avec sa mère dans un parc. C'est la description détaillée de l'atmosphère qui y règne, alors qu'elle admet en même temps ne 
plus arriver "à entendre la voix [de sa mère] en ce temps-là", qui lui vaut les reproches de la deuxième voix: "- Ne te fâche pas, mais ne crois-tu pas que là, avec ces roucoulements, ces piétinements, tu n'as pas pu t'empêcher de placer un petit morceau de préfabriqué..." (Sarraute, 1983: 20-21). Plus loin, si la première voix se laisse emporter par les mots au point de déformer la réalité par des effets de style, la seconde voix, tout en restant amicale, réplique:

“- Fais attention ou tu vas te laisser aller à l'emphase” (Sarraute, 1983: 166). Par conséquent, rien d'artificiel ou de démesuré dans le style, impossible pour la première voix de se "laisser aller". Ailleurs, lorsque la première voix décrit les "façades mornes" qui côtoient sa maison, c'est l'euphémisme que la seconde voix critique et non plus sa déclamation. Aussi rectifiet-elle non sans impudence: “- Mortes, devrais-tu dire, sans avoir peur d'exagérer” (Sarraute, 1983: 113).

La deuxième voix exerce aussi son contrôle sur des comparaisons ou des mots qui ne pouvaient être ceux de l'enfant. "Tu connaissais déjà ces mots...", doute-t-elle lorsque la première voix emploie les mots "insupportable", "fou" et "maniaque" pour décrire l'entêtement de l'enfant à mâcher ses aliments jusqu'à ce qu'ils soient "aussi liquide qu'une soupe" (Sarraute, 1983: 14-15). Un peu plus loin, même scepticisme lorsque la première voix suggère à travers un langage purement métaphorique la bataille livrée par l'enfant pour tenir sa promesse à sa mère: "Je suis toujours là, à mon poste... je résiste... je tiens bon sur ce bout de terrain où j'ai hissé ses couleurs, où j'ai planté son drapeau..." (Sarraute, 1983: 17). La deuxième voix explique que les sentiments ressentis alors, "ne pouvaient pas se former à cet âge-là dans [s]a tête" et encourage la première voix à rectifier: "- Bien sûr que non [...] C'était ressenti, comme toujours, hors des mots, globalement..." (Sarraute, 1983: 15-17). Même rectification dans cet autre exemple, où les comparaisons utilisées par la première voix pour décrire son sentiment de "délivrance" occasionné par un reproche de son oncle, ne pouvaient être celles de l'enfant:

- Il n'est pas possible que tu l'aies perçu ainsi sur le moment...

- Evidemment. Cela ne pouvait pas m'apparaître tel que je le vois à présent, quand je m'oblige à cet effort... dont je n'étais pas capable. (Sarraute, 1983: 86)

Rares sont les cas où le scepticisme de la seconde voix est contesté par une réaffirmation déterminée de la première:

- Tu le sentais vraiment à cet âge?

- Oui [...] Ce sont des choses que les enfants perçoivent mieux encore que les adultes. (Sarraute, 1983: 58)

Ces exemples de prolepse surgissent dès que la première voix quitte le domaine des faits pour analyser la perception que le personnage en a ou pouvait en avoir. Ainsi, lorsque la première voix se souvient des après-midis passés à jouer au Luxembourg, la première voix 
entrevoit le piège préparé par la deuxième voix et refuse de reconstituer la sensation dont le souvenir lui échappe: “Rien ne m'en est resté et ce n'est tout de même pas toi, qui va me pousser à chercher à combler ce trou par un replâtrage" (Sarraute, 1983: 24). Ailleurs, lorsque la première voix évoque le souvenir du départ de l'enfant avec sa mère pour Paris, où elle sera finalement abandonnée à son père, la seconde voix suggère que la détresse éprouvée à ce moment-là par l'enfant était une sorte de "pressentiment", quelque chose qui "l'aurait fait sentir que cette fois ce n'était pas un départ comme les autres". Mais la première voix rejette cette version romancée pour une explication plus rationnelle des faits: “- J'ai peine à croire $[\ldots]$ que déjà à ce moment-là elle ait pu envisager... Non, il n'est pas possible qu'elle ait délibérément voulu me laisser à mon père" (Sarraute, 1983: 108). Dans ces deux derniers exemples les rôles des deux voix se sont exceptionnellement inversés: c'est la première voix qui s'oppose et corrige cette fois la première. Toutes ces interruptions et corrections confirment encore une fois au lecteur les efforts d'objectivité et d'authenticité entrepris par Sarraute pour évoquer son passé.

Après "contrôle", le deuxième rôle que Lejeune identifie pour la seconde voix est "écoute" (Lejeune, 1990: 34). Dans cette nouvelle fonction, la première et la seconde voix entretiennent un rapport comparable à celui qui lie analysé et analysant. Semblable, en effet, au psychothérapeute, la seconde voix encourage la première à faire resurgir certains épisodes de son passé pour qu'ils soient assumés en toute lucidité. "Ce contribut analytique", explique à ce sujet Françoise Van Roey-Roux, "est indispensable au récit autobiographique qui a pour objectif, par définition, de mettre en évidence le fil conducteur d'une existence " (Roey-Roux, 1984: 281). Une fonction qui pourrait également se comparer à la maïeutique, où la deuxième voix chercherait à faire découvrir à la première le savoir qu'elle porte en elle. Plus ou moins forte, cette pression insistante de la seconde voix est suggérée à travers les points de suspension. C'est ce qui arrive dans l'exemple suivant, où la deuxième voix oblige la première à revivre une scène douloureuse: celle où l'enfant, désirant participer au jeu de sa mère et son beau-père qui "faisaient semblant de lutter", se sent "repoussée violemment" (Sarraute, 1983: 74). Tandis que la première voix donne des versions "relativement apaisantes" du passé, la deuxième voix intervient discrètement pour manifester sa perplexité et suggérer une version différente des faits (Lejeune, 1990: 34). En interrompant le récit par une suite de questions, elle parvient, en effet, à extraire de la première voix trois versions qui se suivent en crescendo, jusqu'à l'adjudication finale: "un corps étranger":

- Et c'est tout? Tu n'as rien senti d'autre? [...]

- C'est vrai... je dérangeais leur jeu.

- Allons, fais un effort...

- Je venais m'immiscer... m'insérer là où il n'y avait pour moi aucune place.

- C'est bien, continue...

- J'étais un corps étranger... qui gênait...

- Oui: un corps étranger. Tu ne pouvais pas mieux dire. (Sarraute, 1983: 75) 
Grâce au dialogue et à la seconde voix, qui semble en savoir autant sinon plus que la première sur les sentiments de l'enfant, l'auteur invite le lecteur à explorer la complexité des réactions provoquées chez Natacha par certains gestes ou mots prononcés dans le passé. C'est, en effet, à travers de longues reconstructions comme celle-ci que l'auteur expose le mieux le but de sa recherche entreprise dans Enfance. En obligeant la première voix à quitter le souvenir factuel pour le souvenir émotif souvent pénible à évoquer, la seconde voix accomplit l'objectif même du récit: découvrir "ce qui tremblote quelque part dans les limbes" (Sarraute, 1983: 9). A ce propos, Monique Gosselin remarque: “l'usage de cette seconde parole qu'est l'autre voix donne du relief à ce qui risquerait de n'être qu'un récit plat, unidimensionnel et permet à Nathalie Sarraute de retrouver ce qui a toujours été son domaine de romancière, celui de la "sous-conversation"” (Gosselin, 1996: 237). Un autre exemple d'introspection encouragé par la deuxième voix est celui qui apparaît dans l'épisode où la première voix refuse de donner un nom à l'attitude crispée du père dès que l'enfant lui parle de sa mère. Ce que la première voix devine dans les expressions faciales du père est "quelque chose" que la première voix "ne veu[t] pas voir" (Sarraute, 1983: 127). Les interprétations de la deuxième voix qui cherche, en revanche, à remplacer ce "quelque chose" par "de la rancune, de la réprobation" ou encore "du mépris", sont fermement réfutées:

- Mais je n'appelle pas cela ainsi. Je ne donne à cela aucun nom, je sens confusément que c'est là, en lui, enfoui, comprimé... je ne veux surtout pas que cela se mette à bouger, que cela vienne affleurer... (Sarraute, 1983: 127)

Dans cet exemple, la rectification de la première voix intensifie efficacement l'objectif du récit, soit la recherche d'une expérience ressentie mais indéterminée. Ailleurs, la forme intimidante de l'interrogatif est remplacée par la forme affirmative, plus engageante. En se sentant accompagnée et non plus harcelée par la deuxième voix, la première voix prendra en main plus facilement l'épisode douloureux qu'elle hésite à narrer: “- Mais essaie de te rappeler... il a dû pourtant arriver parfois..." (Sarraute, 1983: 61). Parfois la deuxième voix joue aussi le rôle de tentateur, comme lorsqu'elle incite la première voix à succomber à la tentation d'évoquer un de ces "beaux souvenirs d'enfance" tant redoutés. Malgré les réticences de la première voix qui avoue "hésite[r] un peu...", la seconde voix continue à l'encourager:

- Ça se comprend... une beauté si conforme aux modèles... Mais après tout, pour une fois que tu as cette chance de posséder, toi aussi, de ces souvenirs, laisse-toi aller un peu, tant pis, c'est si tentant... (Sarraute, 1983: 32)

Cette fois-ci la première voix se laisse tenter: le souvenir-cliché étant annoncé et justifié par la seconde voix, la première peut exceptionnellement se "laisser aller". A mesure que le texte avance, la deuxième voix se fait plus envahissante. Puisqu'elle connaît le passé de la première voix aussi bien que cette dernière, son travail consiste parfois à mettre en forme ce 
que la première voix ressent. C'est elle, en effet, qui fait les rapprochements, enchainements, et les déductions qui s'imposent à partir des événements évoqués:

- Il est étrange que ce soit juste cette fois-là que tu aies ressenti pour la première fois une telle détresse au moment de ton départ... On pourrait croire à un pressentiment [...] quelque chose qui t'aurait fait sentir que cette fois ce n'était pas un départ comme les autres... (Sarraute, 1983: 108)

C'est, comme l'explique Lejeune, dans ce troisième et dernier rôle de "collaboration", que la deuxième voix, sans plus s'opposer à la première, prend le relais (Lejeune, 1990: 35). Lorsque, par exemple, la première voix décrit sa réaction devant l'ours que sa petite sœur a "déchiré", la deuxième voix interprète: “- Jamais il ne t'est arrivé d'en vouloir à quelqu'un comme à ce moment-là tu en as voulu à Lili” (Sarraute, 1983: 186). Même chose pour le souvenir de sa rédaction "Mon premier chagrin" où la deuxième voix explique: "Jamais au cours de toute la vie aucun des textes que tu as écrits ne t’a donné un pareil sentiment de satisfaction, de bien-être..." (Sarraute, 1983: 213). Il est rare cependant, explique Lejeune, que le texte s'installe durablement dans un tel régime de symbiose et que les deux voix travaillent ensemble afin de faire resurgir le passé. Cette fonction ne dure souvent que le temps d'une réplique. C'est la fonction "contrôle" qui semble dominer le récit, et c'est à travers elle que Sarraute authentifie tout ce qu'elle écrit. Plus que dans l'enfance, le lecteur est donc plongé dans le procédé de création de Enfance. Non seulement est-il tenu au courant des difficultés qui surgissent dans cette évocation du passé, mais il assiste en même temps à la construction d'un "moi" partagé et constitué par plusieurs voix.

\section{Voix implicites}

Si cet article s'est intéressé, jusqu'ici, au rôle des deux voix narratives et à la forme dialogique, il s'agit maintenant de se tourner vers les voix implicites de Enfance, à savoir la voix de l'enfant, de l'auteur et toutes celles qui ont marqué son passé: les voix de la mère, du père et de la belle-mère, Véra. C'est à la première personne du singulier que sont désignés le narrateur et le personnage. Mais contrairement au "je" du narrateur - ou plutôt des narrateurs puisqu'il s'agit des deux voix du dialogue - qui demeure stable tout au long du récit, le "je" du personnage subit une évolution. Il passe en effet de l'âge de cinq ou six ans à celui de l'entrée au Lycée, soit douze ans. Il arrive parfois que l'enfant, Natacha, soit sensible à cette évolution, qu'elle se rende compte du changement de sa personnalité. C'est le cas, par exemple, lorsqu'elle se souvient à neuf ans de ses "idées", en comparant l'obsession qu'elle en avait à huit ans avec son soulagement présent: "Comment est-il possible que j'aie pu éprouver cela il y a si peu de temps, il y a à peine un an [...] Comme il est délicieux, le contraste avec ce que je suis maintenant..." (Sarraute, 1983: 135). Comme le remarque à ce propos Keling Wei, “l'enfance a ainsi des âges différents, comporte l'évolution et donc la 
superposition des voix" (Wei, 2004: 106). Mais alors que le récit autobiographique utilise traditionnellement le passé pour séparer le "je" narrant du "je" narré, Sarraute utilise essentiellement dans Enfance le présent. Le temps qui sert normalement à distancer le narrateur du personnage, voilà qu'il les confond, les unifie. Aussi, comme le remarque justement Lejeune, "le lecteur aura l'impression d'entendre l'enfant, au point même de croire parfois qu'elle a, passagèrement, pris en charge la fonction narrative" (Lejeune, 1990: 36). C'est le cas, en effet, de tous ces débuts de chapitre, où l'instance narrative raconte au présent de l'énonciation une vision passée du "je" enfant: "Je suis assise près de maman dans une voiture fermée tirée par un cheval, nous cahotons sur une route poussiéreuse" (Sarraute, 1983: 31). Tout ceci a pour effet de réactualiser les faits passés et de donner l'impression d'une continuité entre l'enfant et l'adulte. Une permanence attestée aussi par le recours aux locutions verbales "ce qui m'est resté" ou "ce qui a subsisté", qui mettent l'accent sur la survie de sensations éprouvées il y a longtemps (Sarraute, 1983: 56-57). Un procédé employé également dans cet autre dialogue autobiographique qu'est Une autobiographie allemande où l'amitié et la confiance entre Hélène Cixous et Cécile Wajsbrot ont permis à cette seconde de poser des questions et à la première d'y répondre sans fausse pudeur. Un dialogue qui aborde identité, mémoire, langues et qui partage avec Nathalie Sarraute un même effort de retrouver des sensations, impressions du passé et emploie le temps présent pour désigner son “je” d'antan: "Je me vois avec Omi dans les rues de Köln [...] A l'hôtel des Bains de Bad Nauheim, je m'ennuie [...] J'ai quatorze ans" (Cixous \& Wajsbrot, 2016: 24). Un passage qui fait aussi écho aux premières pages de L'Amant où l'emploi du présent provoque un contraste étonnant par la juxtaposition du "visage détruit" de Marguerite Duras et son image de jeune fille de quinze ans: "J'ai un visage lacéré de rides sèches et profondes, à la peau cassée [...] J'ai un visage détruit. Que je vous dise encore, j'ai quinze ans et demi" (Duras, 1984: 10-11).

Si la première personne désigne le narrateur et le personnage, elle est aussi la manifestation de l'auteur. C'est l'instance narrative, par exemple, pour expliquer la raison pour laquelle il lui arrive parfois de raconter l'épisode de son premier "roman":

- C'est un des rares moments de ton enfance dont il t'est arrivé parfois, bien plus tard, de parler...

- Oui, pour répondre, pour donner des raisons à ceux qui me demandaient pourquoi j'ai tant attendu avant de commencer à "écrire"... (Sarraute, 1983: 85)

Cependant, c'est surtout implicitement que l'instance narrative intervient dans la narration, comme lorsqu'elle invite le lecteur à découvrir la "mise en forme" ou récupération d'un moment particulier survenu dans les Jardins du Luxembourg et lui faire saisir le décalage entre expérience et langage. Il ne s'agit pas pour elle d'atteindre une définition précise de la sensation ressentie mais plutôt d'évoquer des alternatives capables de "recueillir" la réalité intérieure d'un état passé: 
Quel mot peut s'en saisir? pas le mot à tout dire "bonheur", qui se présente le premier, non, pas lui... "félicité", "exaltation", sont trop laids, qu'ils n'y touchent pas... et "extase"... comme devant ce mot ce qui est là se rétracte... "Joie", oui, peut-être... ce petit mot modeste, tout simple, peut effleurer sans grand danger... mais il n'est pas capable de recueillir ce qui m'emplit. (Sarraute, 1983: 67)

Suggestions, alternatives et corrections cherchent l'expression adéquate pour définir une intense sensation de vie dont l'auteur souligne la pureté et son caractère irréversible:

Jamais plus cette sorte d'intensité-là, pour rien, parce que c'est là, parce que je suis dans cela, dans le petit mur rose, les fleurs des espaliers, des arbres, la pelouse, l'air qui vibre... je suis en eux sans rien de plus, rien qui ne soit à eux, rien à moi (Sarraute, 1983: 67).

On se retrouve à la fin du texte d'une manière impossible ("plus jamais", "encore maintenant"), dans un lieu au-delà du langage et de ses dangers, mais ceci grâce à et à travers le langage. Comme l'explique justement P.J. Eakin, le passage retrace simultanément deux drames: celui du passé et celui de sa recréation (Eakin, 1992: 39) ${ }^{4}$. Même chose pour cet autre passage où Sarraute cherche à récupérer la magnifique image qui lui reste d'Ivanovo. Ici encore, son manque de confiance face aux mots la fait hésiter: "J'ai envie de la palper [...] de la parcourir avec des mots, mais pas trop fort, j'ai si peur de l'abîmer..." (Sarraute, 42). L'acte de remémorer le passé est ainsi perçu dans toute sa fragilité, comme un travail de récupération presque tactile.

Après la voix de l'enfant, et celle implicite de l'instance narrative, c'est l'image et surtout la voix de la mère qui dominent la première partie de Enfance. Mais comme le constate Simone Benmussa, sa figure reste élusive, un ensemble de perceptions, de paroles et ses longs échos:

Il n'y a jamais en effet de description directe des personnages, pas de description psychologique ou morale de la mère, pas de jugements, pas de critique, mais seulement ce qui vient d'elle, ce qu'elle impose, comment elle est perçue, indirectement, par la petite fille, par le rapport qui s'établit entre elles. (Benmussa, 1984: 80)

C'est la mère qui définit le comportement à adopter chez l'enfant, et c'est à travers ses phrases que celle-ci apprend son rôle par rapport à celui des grands. L'influence des mots de la mère sur l'enfant se manifeste très clairement dans la deuxième scène de Enfance où Natacha s'obstine à "mâcher les aliments jusqu'à ce qu'ils deviennent aussi liquides qu'une soupe" (Sarraute, 1983: 15). Ces mots, prononcés par un docteur de Paris, sont ceux que la mère "recommande" à Natacha de ne pas oublier. En son absence, ces mots remplacent la mère et deviennent des paroles véritablement "sacrées" que l'enfant s'efforce fidèlement à respecter:

4 "The passage records two dramas at once, that of the past itself and that of its recreation". 
Ma mère [...] n'est pas ici, elle m'a fait emporter cela avec moi... 'aussi liquide qu'une soupe' c'est d'elle que je l'ai reçu... elle me l'a donné à garder, je dois le conserver pieusement, le préserver de toute atteinte. (Sarraute, 1983: 17-18)

Même enracinement de mots de la mère chez Julien Green qui, dans Partir avant le jour, se souvient que la façon dont sa mère prononçait le mot "body" était si chargée de dégoût que "jusqu'à l'âge de quinze ou seize ans" il hésitait à prononcer le mot à son tour, comme s'il désignait "une chose honteuse" (Green, 1963: 85). Mais ce que Natacha retient surtout de sa mère, ce n'est pas seulement le caractère "sacré" de certains mots qu'elle prononce, mais l'indifférence et la cruauté de certaines de ses phrases. Comment ne pas se sentir affligée en écoutant accidentellement sa mère se lamenter: "Quand je pense que je suis restée ici enfermée avec Natacha pendant tout ce temps sans que personne ne songe à me remplacer auprès d'elle". Dans cet exemple, la première voix avoue ne plus pouvoir se souvenir de ce qu'elle ressentit à l'époque mais le fait qu'elle se souvienne encore maintenant de ces paroles prouve le contraire. C'est ce que lui suggère sournoisement la seconde voix en interrompant son discours par un jeu de mots suggestif: "Ce que j'ai ressenti à ce moment-là s'est vite effacé... - S'est enfoncé, plutôt...” (Sarraute, 1983: 40). L'émotion éprouvée par l'auteur est donc encore toute "tiède, battante", comme dirait Marguerite Duras au sujet de L'Amant et de La Douleur (Duras, 1987: 89).

Ce sont surtout les "dictons" prononcés par sa mère que Sarraute retranscrit dans Enfance, comme celui que la mère proclame sévèrement lorsqu'elle entend sa fille dire à son ours en peluche qu'elle part à Paris voir son père et "une autre maman": "Tu n'as au monde qu'une seule maman" (Sarraute, 1983: 104). Ailleurs, lorsque l'enfant demande la permission à sa mère d'appeler sa belle-mère "maman-Véra", elle reçoit une lettre blessante et amère qui explique clairement que "ce nom, maman, ne peut s'accoler à aucun autre" (Sarraute, 1983: 219). Les énoncés de la mère sont donc d'emblée définitifs, et c'est le plus souvent sous la forme abstraite de l'axiome et de la sentence que la parole maternelle est transmise: “Un enfant qui aime sa mère trouve que personne n'est plus beau qu'elle" (Sarraute, 1983: 95). Une pseudo-loi que l'enfant emporte au fond d'elle-même comme un paquet bien enveloppé et qu'elle n'ouvrira qu'une fois rentrée, "pour voir ce qu'il contient” (Sarraute, 1983: 95). Même terreur verbale chez la mère de Simone de Beauvoir dans Mémoires d'une jeune fille rangée, où le moindre "reproche" ou "froncement[s] de sourcil[s]" constitue chez la jeune protagoniste une menace de néantisation:

Tout reproche de ma mère, le moindre de ses froncements de sourcils, mettait en jeu ma sécurité: privée de son approbation, je ne me sentais plus le droit d'exister. (De Beauvoir, 1958: 56)

Une angoisse d'extinction renforcée chez la mère de Sarraute par son pouvoir de concrétiser ses référents. C'est ainsi qu'en transgressant la défense: "si tu touches à un poteau 
comme celui-là, tu meurs...", l'enfant se croit effectivement morte (Sarraute, 1983: 27). Le temps altère si peu les paroles de la mère que l'adulte a l'impression de ressentir leur choc avec presque autant de force et intensité qu'au moment où elles ont été prononcées: "Jamais aucune parole, si puissamment lancée qu'elle fut, n'a eu en tombant en moi la force de percussion de certaines des siennes" (Sarraute, 1983: 27). Une "force" des mots maternels partagée encore une fois par la jeune Simone de Beauvoir, qui se souvient de l'expression "C'est ridicule!" et de son pouvoir "paralysant": "Dirigé contre nous, il [ce verdict] nous précipitait de l'empyrée familial dans les bas-fonds où croupissait le reste du genre humain" (de Beauvoir, 1958: 57). Parfois le sens des paroles maternelles revêt l'intensité de la représentation visuelle. Aussi les mots "Véra est bête", confiés un jour par la mère à Natacha, sont si chargés de signifiés que l'enfant ne peut s'empêcher de voir à la place de sa belle-mère "une grande personne affublée d'un bonnet d'âne" (Sarraute, 1983: 188-189). Semblable à Roland Barthes qui, dans un fragment de son Roland Barthes par Roland Barthes, avoue: "J'ai une maladie: je vois le langage" (Barthes, 1975: 164), Sarraute dérive aussi 1'“écoute" en "scopie". Pour Barthes, le passage s'opère ainsi:

Ce que je devrais simplement écouter, une drôle de pulsion, perverse en ce que le désir s'y trompe d'objet, me le révèle comme une 'vision' [...] A la scène primitive, où j'écoute sans voir, succède une scène perverse, où j'imagine voir ce que j'écoute. L'écoute dérive en scopie: du langage, je me sens visionnaire et voyeur. (Barthes, 1975: 164)

Ailleurs, c'est l'adjectif “intelligent” avec lequel la mère qualifie le père qui restera ancré dans la mémoire de l'enfant (Sarraute, 1983: 194). Désormais, tout ce que Véra et le père diront ou feront sera toujours jugé par l'enfant sous cette lumière. Une autre remarque cruelle qui bouleversera Natacha est la phrase "femme et mari sont un même parti" où la sensation éprouvée alors par l'enfant est celle d'“un corps étranger" (Sarraute, 1983: 75). Un sentiment d'exclusion, d'abandon semblable à celui qu'elle ressent lorsque sa mère lui raconte des histoires avec “cette impression que plus qu'à [elle] c'est à quelqu'un d'autre qu'elle raconte" (Sarraute, 1983: 20); ou encore l'indignation, exprimée bien plus tard, que les lettres que lui adresse sa mère deviennent progressivement plus lointaines et semblent se diriger "à un petit enfant": "A qui croit-elle raconter [...] Elle ne sait pas qui je suis maintenant, elle a même oublié qui j’étais" (Sarraute, 1983: 126). Vers la fin du récit le manque de communication entre mère et enfant est encore plus flagrant. Lorsque, après deux ans et demi de séparation, la mère rentre à Paris voir sa fille, elles se trouvent toutes les deux dans l'embarras: "Nous restons là l'une en face de l'autre, nous nous regardons, je ne sais pas quoi lui dire et je vois que maman ne sait pas très bien quoi dire non plus..." (Sarraute, 1983: 252). Incapable de trouver les mots justes, appropriés, la mère est perdue, elle ne sait plus très bien à qui elle parle, si à un enfant ou à un adulte. Et Natacha sent pour la première fois "l'indifférence" à l'égard de sa mère (Sarraute, 1983: 255). Une distance qui culmine 
un peu plus loin, lorsque l'enfant décide d'aller à Versailles avec Véra, juste après l'arrivée de sa mère à Paris. Indignée, la mère repart en Russie et les derniers mots qu'elle adresse à sa fille, un pur exemple de "narcissisme verbal", comme dirait Valérie Minogue, confirment encore une fois un refus de communication directe et personnelle avec l'enfant (Minogue, 1988: 222):

Ecoute comme il est beau en russe, le mot 'gniev', et comme en français 'courroux' est beau... c'est difficile de dire lequel a plus de force, plus de noblesse... elle répète avec une sorte de bonheur 'Gniev'... 'Courroux'... elle écoute, elle hoche la tête... Dieu que c’est beau... et je réponds Oui. (Sarraute, 1983: 258)

Plus discrète est la voix du père. Si son image est présente tout au long du récit, ses paroles n'ont pas la même répercussion qu'ont celles de la mère ou de la belle-mère Véra sur l'enfant. Les rapports qu'entretiennent père et fille étant moins conflictuels, ils fournissent, comme le suggère Michael Sheringham, moins de prétexte à ce qu'il nomme "incidents autobiographiques", à savoir un événement mineur auquel le discours de l'autobiographe attribue une lourde charge de sens (Sheringham, 1993: 102) ${ }^{5}$. Ce sont souvent des paroles douces et rassurantes que l'auteur retient de son père, ou sa "voix basse et incertaine, comme un peu éraillée" lorsqu'il se met à chanter une "vieille berceuse". "Il ne sait pas bien chanter", se souvient la voix narrative, mais "cette maladresse donne à ce qu'il chante quelque chose d'encore plus touchant" (Sarraute, 1983: 52). Les expressions "ne t'en fais pas" ou encore "tu verras, dans la vie, tôt ou tard, tout s'arrange", employées pour réconforter Natacha après la "trahison" de sa mère, sont encore un exemple de l'amour un peu maladroit que le père porte à son enfant. Parfois c'est l'enfant qui assume le rôle d'émetteur et lancerait à son père certains mots "catalyseurs", capables, comme le suggère Simone Benmussa, de développer "des mouvements, des tropismes représentant une certaine agitation de la conscience ou du subconscient" (Benmussa, 1987: 168). C'est le cas des mots "tu m'aimes" ou "je t'aime" que Natacha prononce avec précaution, en contrôlant le ton de sa voix, sachant que son père "déteste trop ce genre de mots":

Je savais que ces mots 'tu m'aimes', 'je t'aime' étaient de ceux qui le feraient se rétracter, feraient reculer, se terrer encore plus loin au fond de lui ce qui était enfoui. (Sarraute, 1983: 58)

La réponse du père: "Mais oui, mon petit bêta, je t'aime", et l'italique rajouté ici par l'auteur, suggèrent son ton malicieux et délivrent père et enfant des contraintes du langage, de "ces mots ridicules, indécents". Natacha est satisfaite et avoue: “j'ai pu le taquiner un peu et puis le rassurer" (Sarraute, 1983: 59). Abandonnée et trahie par sa mère qui rompt le pacte secret qui la lie à sa fille, Natacha noue à partir de là avec son père un "lien si invisible que

5 "A minor event to which the autobiographer's discourse ascribes a heavy payload of meaning". 
rien n'a pu détruire" (Sarraute, 1983: 116). Lorsque plus tard le père soigne Natacha d'une infection contractée à la suite d'une vaccination, il assume totalement le rôle maternel et ce sont les diminutifs "Tachotchek, ma petite fille, ma chérie" qui témoignent de son attachement (Sarraute, 1983: 225). Un lien encore plus fort entre père et enfant se produit à la suite d'une lettre où la mère, d'un ton blessant, rejette encore une fois sa fille en déclarant: "Je vous félicite, vous avez réussi à faire de Natacha un monstre d'égoïsme. Je vous la laisse" (Sarraute, 1983: 258). A partir de là, ce ne sont plus les diminutifs "Tachok" ou "Tachotchek" que le père emploie pour désigner son enfant, mais les mots "ma fille, ma petite fille, mon enfant" où le possessif suggère ce "lien à part" qui les unit, "comme l'assurance de son soutien, et aussi un peu comme un défi" (Sarraute, 1983: 270).

L'autre personne à laquelle l'enfant porte une attention particulière est sa belle-mère Véra. Comme la mère et le père, Véra est caractérisée essentiellement par son discours. C'est sa voix que le lecteur entend à travers les voix des narratrices et du personnage. Mais ce sont aussi les discours que les autres portent sur elle, en particulier le discours de la mère, qui définissent Véra. Les mots "Véra est bête" (Sarraute, 1983: 189) ou encore "Cette... Véra n'est pas tout à fait normale... il paraît que c'est une hystérique...” (Sarraute, 1983: 255), sont des images qui lui collent et contre lesquelles Natacha cherchera à s'opposer. Aussi lorsqu'un jour Natacha demande à sa belle-mère si elles vont bientôt rentrer à la maison, et que celleci répond sèchement: "Ce n'est pas ta maison", c'est avec précaution et une certaine dose d'incrédulité qu'est traitée cette réponse (Sarraute, 1983: 130). Ce qu'elle risquerait en effet est de transformer Véra et Natacha en "personnages de contes de fées" de la "méchante marâtre" et de "Cendrillon". Si Véra, avoue la seconde voix, a pourtant cet effet "par moments, de décoller du réel, de s'envoler dans la fiction" (Sarraute, 1983: 130), "aucun mot", explique plus tard la première voix, "ne vient s'appliquer sur elle" (Sarraute, 1983: 191). Aussi, lorsque les autres enfants qualifient Véra de "méchante", Natacha avoue: "Cela me surprenait... aussitôt surgissaient des images qui ne trouvaient pas de place dans "méchante"" (Sarraute, 1983: 191). La silhouette de la "marâtre" disparaît le soir où Natacha la surprend en train de pleurer. A partir de là, toute comparaison avec ce personnage de conte de fées devient impossible: "Et pour la première fois, j'ai vu quelqu'un d'aussi familier, bien visible, connu que l'était pour moi Véra, devenir sous mes yeux quelqu'un de tout autre" (Sarraute, 1983: 203). C'est alors que "des images, des bribes de récits qui étaient passés comme à distance d'elle" viennent se superposer à celles que Natacha a toujours conservé. Comme suggère Viviane Forrester, il n'existe "plus de marâtre intégrale": "le mot "marâtre" ne peut, seul, définir une femme" (Forrester, 1983: 21). La dernière image de Véra viendra contredire encore une fois ces dernières impressions puisqu'elle est celle de la nurse anglaise, rencontrée par l'auteur vingt ans après et qui avoue: "I still see your step-mother in my nightmares" (Sarraute, 1983: 264).

Ce que toutes ces différentes évocations suggèrent, c'est que les personnes décrites 
dans Enfance ne se limitent pas à une seule image. Les identités doivent en effet rester aussi intactes que les souvenirs évoqués et ne pas être contaminées par la critique ou le jugement. Aussi l'auteur démonte-t-elle subtilement tous les personnages romanesques qui risqueraient de se créer, comme cette image de "babouchka", la mère de Véra:

Il est impossible de la modeler en une mignonne statuette bleue et rose de grand-mère de conte de fées... impossible de la figer... il y a quelque chose en elle de toujours mouvant, de pétillant, quelque chose de vif. (Sarraute, 1983: 227)

Ce que Sarraute cherche, par conséquent, à éviter est de transformer son entourage en personnages. Comme le constate justement Monique Gosselin, il s'agit d'une défiance "très sarrautienne":

Elle redoute [...] les événements ou les crises qui donnent au personnage une consistance, une épaisseur que la vie lui refuse parce qu'elle se dissout en menus incidents sans relief, en gestes timides à peine esquissés qui ne forment, mis bout à bout, ni une histoire ni un récit. (Gosselin, 1996: 89)

\section{Voix vivantes}

C'est parce que les mots apparaissent comme des éléments vivants et souvent hostiles que l'enfant est toujours très attentive à leur prononciation et variations de ton. Non seulement chaque voix retranscrite dans Enfance possède-t-elle sa singulière intonation, mais elle est aussi intimement liée à ce croisement de russe, français, allemand, anglais qui ont accompagné successivement Tachok, Tachotchek, Natacha. Dans la première scène c'est le ton "sifflant, féroce" du verbe allemand "zerreissen" qui assiste le désir de transgression de l'enfant. Ailleurs, c'est la douceur du russe que Sarraute évoque à travers la voix "grave, à peine un peu rauque" de sa mère et sa "prononciation où seulement le ' $r$ ' roulé et une certaine intonation révèlent l'accent russe" (Sarraute, 1983: 250-251). Puis il y a l'anglais, une langue étrangère qui, explique l'auteur, "par elle-même m'enchantait" (Sarraute, 1983: 262). Une sensibilité aux sons, tonalités et langues comparable encore à celle que ressent Cixous où l'auteur avoue: "Mais il y a aussi le charme de la langue: j'ai besoin d'entendre mes sujets, chaque phrase qui est une production de l'âme" (Cixous \& Wajsbrot, 2016: 51). Aussi se réjouit-elle de retrouver "le timbre, la cadence, la voix" de sa mère dans un petit "Wortwitz" allemand prononcé lorsqu'elle était enfant. Dans Enfance il s'agit de la phrase "Parce que ça ne se fait pas", prononcée en français par Véra lorsque Natacha lui demande "Pourquoi on ne peut pas faire ça?" qui laisse entrevoir son caractère "buté":

De son ton buté, ferme, en comprimant les voyelles encore plus qu'elle ne le fait d'ordinaire, les consonnes cognées les unes contre les autres s'abattent, un jet dur et dru qui lapide ce qui en moi remue, veut se soulever [...] (Sarraute, 1983: 187) 
Pour mieux atteindre l'enfant, Véra aura parfois recours au russe lorsque cette langue est plus expressive que le français. C'est le cas du verbe russe "podbrossili" qu'elle lance à Natacha pour lui annoncer que sa mère l'abandonne:

Les mots russes ont jailli durs et drus comme ils sortaient toujours de sa bouche... "podbrossili" un verbe qui littéralement signifie "jeter", mais qui a de plus un préfixe irremplaçable qui veut dire "sous", "par en dessous" et cet ensemble, ce verbe et son préfixe, évoque un fardeau dont subrepticement on s'est débarrassé sur quelqu'un d'autre [...] (Sarraute, 1983: 182)

Si cet épisode semble figurer comme un "magnifique traumatisme d'enfance", l'apparente brutalité des mots apaise en même temps l'enfant du douloureux sentiment de culpabilité engendré par les reproches de la mère et ceux de Véra. L'enfant conclue en effet:

On ne veut pas de moi là-bas, on me rejette, ce n'est donc pas ma faute, ce n'est pas de moi qu'est venue la décision, je dois rester ici que je le veuille ou non, je n'ai pas le choix. (Sarraute, 1983: 184)

Le "on t'a abandonnée" procure par conséquent un sentiment de délivrance douloureuse mais bénéfique. Une autre description classique d"'enfance malheureuse" qui menace d'envahir le récit de clichés est l'exclamation "Quel malheur !", prononcée par la bonne: Le mot "malheur", de même que son antonyme "bonheur", ou l'expression "beaux souvenirs" qui contraste avec les "traumatismes d'enfance", sont tous des termes qui renvoient au "tout cuit", à un ensemble d'idées toutes faites que l'auteur prend soin de mettre entre guillemets, comme pour s'en écarter: 'Le 'malheur' qui ne m'avait jamais approchée, jamais effleurée, s'est abattu sur moi. Cette femme le voit. Je suis dedans. Dans le malheur" (Sarraute, 1983: 121). C'est par conséquent avec révolte que l'enfant se dégage de ces mots opprimants: “Je ne resterai pas dans ça, ou cette femme m'a enfermée...” (Sarraute, 1983: 122).

Semblables à ces mots qui "s'abattent sur vous et vous enferment", les "idées" que Natacha forme dans sa tête l'emprisonnent dans un monde effrayant de pensées indépendantes (Sarraute, 1983: 122). Aussi n'ose-t-elle pas les exprimer, et lorsqu'on lui demande ce qu'elle a, elle répond "J'ai mes idées", un peu comme on répondrait "J'ai mes douleurs. J'ai ma migraine", mais avec la différence que ses "idées" sont "un mal honteux", qui ne peuvent être confiées (Sarraute, 1983: 101). Inutile de chercher à refouler une idée car plus Natacha la repousse, plus l'idée "augmente encore sa poussée" (Sarraute, 1983: 98). Un exemple d'"idée" est celle qui suggère que la poupée de la vitrine d'une maison de coiffure est "plus belle que maman". Dépassée par l'angoisse et le chagrin, Natacha partage son idée avec sa mère en espérant que celle-ci finira par la soulager. Mais sa mère lui répond impitoyablement: "un enfant qui aime sa mère trouve que personne n'est plus beau qu'elle" (Sarraute, 1983: 95). Exclue de cette catégorie d'enfants qui aiment leur mère, Natacha se sent aliénée, 
rejetée dans l'anormalité. La conclusion est évidente: elle est un "monstre" (Sarraute, 1983: 97). Une "idée" encore plus forte encore que celle qui l'a provoquée et qui remplit l'enfant d'un sentiment de culpabilité intolérable. Même aliénation et sentiment d'exclusion dans cette sentence prononcée cette fois-ci par Véra: "comment peut-on détester un enfant", qui fait suite à la question de Natacha: "Dis-moi, est-ce que tu me détestes?" (Sarraute, 1983: 272-273). En refusant de personnaliser la phrase et choisissant, au contraire, de s'identifier à travers le pronom personnel indéfini “on", Véra évite de répondre directement à la question posée. Semblable à cette autre généralité énoncée par la mère d'André Gorz dans son autobiographie Le Traître, "une mère aime toujours ses enfants" (Gorz, 1958: 153), la phrase prononcée par Véra est une démonstration de force. En gommant sexe et individualité ces deux mères imposent aux enfants le rôle d'enfant et les oblige à s'intégrer à cette catégorie que Sarraute définit de "pitoyables pygmées aux gestes peu conscients, désordonnés, aux cerveaux encore informes", pour se sentir aimés (Sarraute, 1983: 274). Mais c'est aussi une "catégorie" dont Sarraute et Gorz doivent se détacher afin de revendiquer leur identité et se dégager de la servitude imposée par le monde adulte.

Le langage est donc ressenti comme quelque chose qui déforme et aliène. Contrairement au jeune Sartre qui, dans Les Mots, voit "les mots" comme des "petits reîtres noirs et véloces" (Sartre, 1964: 150) qu'il a l'impression de dominer, Natacha les voient comme des choses vivantes qui semblent la dominer. C'est lors de la récitation d'un poème à un mariage que Natacha éprouve son premier sentiment d'aliénation. Possédée et rendue esclave à travers le langage, l'enfant souffre d'une intense sensation d'humiliation: "Je perçois parfaitement combien est fausse, ridicule, cette imitation de l'innocence, de la naïveté d'un petit enfant" (Sarraute, 1983: 62). En jouant le rôle de l'enfant, Natacha sent qu'elle parcourt "jusqu'au bout ce chemin de la soumission, de l'abject renoncement à ce qu'on se sent être" (Sarraute, 1983: 63). Un travestissement qui contraste fortement avec son assurance lors de ses récitations en classe. Natacha est alors maitre et non plus esclave de son langage (Sarraute, 1983: 180). Même assurance à l'écrit: tel sujet de rédaction à l'école "Votre premier chagrin" représente pour Natacha un "sujet en or" car les mots ne revêtent plus un caractère intimidant, comme dans l'écriture de son premier "roman", mais sont contrôlés et leurs effets attentivement calculés:

Les mots parmi lesquels je me suis posée ne sont pas mes mots de tous les jours, des mots grisâtres, à peine visibles, assez débraillés... ces mots-ci sont comme revêtus de beaux vêtements, d'habits de fête... (Sarraute, 1983: 210)

Ce refuge de l'enfant, dans le monde des surfaces bien polies de la convention et du stéréotype, figurent comme un détour ironique face au monde mobile et incertain de l'écrivain. Mais ce sont déjà une même "liberté" de ne "livrer rien de ce qui n'est qu'à elle" et un désir de récuser le rapport autobiographique qui sont aussi suggérés (Sarraute, 1983: 208). 
C'est donc à l'école que Natacha acquiert une "complète et définitive indépendance" vis-àvis des mots et de ses “idées". Désormais, "ses" idées autrefois incontrôlables sont devenues “des" idées qu'elle peut convoquer sans craindre de se sentir emprisonnée. Aussi, la phrase "Papa a mauvais caractère" s'avère-t-elle inoffensive: "Je l'ai pensé et cela n'appartient qu'à moi. Je n'ai à en rendre compte à personne”, avoue-t-elle assurée (Sarraute, 1983: 136). C'est elle qui contrôle désormais ses idées et qui décide de "les retenir, de les faire rester le temps qu'il faut quand il [lui] arrive d'avoir envie de les examiner avant de les congédier" (Sarraute, 1983: 136). Une domination du langage qui s'accompagne aussi d'une "complète et définitive indépendance" (Sarraute, 1983: 137) par rapport à autrui puisque, comme l'explique Emile Benveniste, c'est "dans et par le langage que l'homme se constitue comme sujet" (Benveniste, 1966: 259). "Tout ce récit d'enfance peut donc être lu”, explique Monique Gosselin, "comme la quête de tropismes à travers lesquels l'enfant se constitue en sujet autonome face aux adultes, et parfois contre eux" (Gosselin, 1996: 84).

\section{Conclusion}

Ce n'est pas seulement l'enfant, Natacha, qui se constitue en sujet dans Enfance, c'est aussi l'auteur qui, à travers les deux "voix" cherche à reconstituer son "moi". Secondée par une deuxième voix qui active le processus de remémoration, la première voix parvient à récupérer, reconstituer certaines sensations et émotions de son "moi" passé. Le terme "restituer”, explique Monique Gosselin, “implique qu'il ne s'agit pas de livrer une pure spontanéité mais de restaurer le passé et ce, par un travail au présent de l'écriture", autrement dit, par "le fruit de tentatives laborieuses et réitérées" (Gosselin, 1996: 39). Comme le constate par conséquent Lejeune:

Enfance est le premier livre de Sarraute dans lequel le moi, la construction du moi, apparaissent explicitement comme des valeurs. Le premier qui offre au lecteur une identification positive. Il apparaît comme l'envers (mais peut-être aussi le fondement secret) de tous ces autres livres. (Lejeune, 1998: 262)

Grâce au dialogue, c'est un progrès de la conscience, un travail de construction du "moi", que Enfance met en scène à travers ces deux voix qui cherchent à récupérer certains "mouvements" et "moments" du passé et à créer un langage capable de les évoquer. Il s'agit en effet pour Sarraute d'illustrer à travers le dialogue une recherche où le "tu", semblable au "meneur de jeu" dans la recherche philosophique par discussion, conduit son interlocuteur "je" à découvrir le savoir qu'elle porte en elle. Le “je” n'est donc plus quelqu'un qui possède la parole comme un bien inaliénable, comme une faculté innée qu'il se contente d'exercer, mais quelqu'un à qui le "tu" donne la parole, pour raconter ce quelque chose qu'il ne connaît pas, ou du moins pas encore au niveau du langage. Témoin discret de cette naissance du langage, 
le lecteur assiste, d'après Lejeune, "à un discours qui lui est destiné, même s'il ne lui est pas directement adressé” (Lejeune, 1980: 36). C'est à lui, en effet, que Sarraute assigne la tâche de poursuivre le travail d'interprétation et de construction de son moi.

\section{Referencias bibliográficas}

Alazet, Bernard. 1990. "Nathalie Sarraute à Cerisy-la-Salle" in Revue des Sciences Humaines, 55 (i), 163-166.

Armel, Aliette. 1990. "Le jeu autobiographique" in Magazine Littéraire, $\mathrm{n}^{\circ}$ 278, 28-31.

Avendaño Anguita, Lina. 2014. "Au gré du regard (Enfance de Nathalie Sarraute)" in Thélème. Revista Complutense de Estudios Franceses, vol. 29, nº 1, 9-22.

BARTHES, Roland. 1966. "Introduction à l'analyse structurale des récits" in Communication, $\mathrm{n}^{\circ} 8,1-27$.

Barthes, Roland. 1975. Roland Barthes par Roland Barthes. Paris, Edition du Seuil (coll. Écrivains de toujours).

Beauvoir, Simone de. 1958. Mémoires d'une jeune fille rangée. Paris, Gallimard.

Benmussa, Simone. 1984. "Les paroles vives" in L'Arc, 95, 76-82.

Benmussa, Simone. 1987. Nathalie Sarraute, qui êtes-vous? Lyon, La Manufacture.

Benveniste, Emile. 1966. Problèmes de linguistique générale. Paris, Gallimard.

Butor, Michel. 1992. "L'usage des pronoms personnels dans le roman" in Essais sur le roman. Paris, Gallimard, 73-88.

Cixous, Hélène \& Cécile Wajsbrot. 2016. Une autobiographie allemande. Paris, Éditions Christian Bourgois.

Duras, Marguerite. 1984. L’Amant. Paris, Éditions de Minuit.

Duras, Marguerite. 1987. La vie matérielle: Marguerite Duras parle à Jérôme Beaujour. Paris, P.O.L.

EAKIn, Paul John. 1992. "The Referential Aesthetic of Autobiography" in Touching the World: reference in autobiography. Princeton, Princeton University Press, 29-39.

Forrester, Vivianne. 1983. "Portrait de Nathalie" in Le Magazine Littéraire, n 196, 18-24.

Gorz, André. 1958. Le Traître. Paris, Éditions du Seuil.

Gosselin, Monique. 1991. "Enfance de Nathalie Sarraute: les mots de la mère" in Revue des Sciences Humaines, 56 (ii), 121-142.

Gosselin, Monique. 1996. Monique Gosselin présente Enfance de Nathalie Sarraute. Paris, Gallimard. 
Gratton, Johnnie. 1995. "Towards narrativity: Nathalie Sarraute's Enfance" in Forum of Modern Languages Studies, 31, 300-311.

Green, Julien. 1963. Partir avant le jour. Paris, Bernard Grasset.

Guerrero, Marisa. 1993. "Las infancias de Nathalie Sarraute (A propósito de Enfance) (I): Pacto autotextual vs. Pacto autobiográfico" in Revista de Filología Francesa, 4, 89-99.

Guerrero, Marisa. 1995. "Las infancias de Nathalie Sarraute, II (A propósito de Enfance): El texto diverso" in Revista de Filología Francesa, 6, 133-145.

JefFerson, Ann. 1990. “Autobiography as intertext: Barthes, Sarraute, Robbe-Grillet”, Intertextuality: theories and practice. Manchester University Press, 108-129.

Keller, Deborah. 2004. “Autobiographie et authenticité chez Nathalie Sarraute" in Le 'Nouveau Roman'en questions, 5, Une 'Nouvelle autobiographie’? Paris, Minard, 57-74.

Lecarme, Jacques \& Lecarme-Tabone, Eliane. 1997. L'Autobiographie. Paris, Armand Colin/Masson.

Lejeune, Philippe. 1975. Le pacte autobiographique. Paris, Editions du Seuil (coll. Poétique).

LeJeune, Philippe. 1980. Je est un autre: l'autobiographie de la littérature aux médias. Paris, Editions du Seuil (coll. Poétique).

Lejeune, Philippe. 1990. "Paroles d'enfance” in Revue des Sciences Humaines, 55 (i), 23-38.

Lejeune, Philippe. 1998. Brouillons de soi. Paris, Editions du Seuil.

Minogue, Valérie. 1986. "Fragments of a childhood: Nathalie Sarraute's Enfance" in Romance Studies, 9, 71-83.

Minogue, Valérie. 1988. "Nathalie Sarraute's Enfance: from experience of language to the language of experience" in Studies in French fiction in honour of Vivienne Mylne, Robert Gibson (ed.). London, Grant \& Cutler, 209-224.

O’Callaghan, Raylene. 1989. “Reading Nathalie Sarraute’s Enfance: reflections on critical validity" in Romanic Review, 80 (3), 445-461.

Ramsey, Raylene L. 1996. "Voice(s) in Nathalie Sarraute's Enfance and the sex of the text" in The French New Autobiographies. University Press of Florida, 117-140.

Rykner, Arnaud. 1991. Nathalie Sarraute. Les Contemporains. Paris, Seuil.

RYKNer, Arnaud. 1992. "Narcisse et les mots-miroirs (Sartre, Leiris, Sarraute autobiographes)" in The Romanic Review, vol. 83, 81-93.

Sarraute, Nathalie. 1983. Enfance. Paris, Gallimard.

SARTre, Jean-Paul. 1964. Les Mots. Paris, Gallimard.

Sheringham, Michael. 1993. French Autobiography: Devices and Desires. Rousseau to Perec. Oxford, Clarendon Press. 
Anales de Filología Francesa, n. ${ }^{\circ}$ 29, 2021

ANTONELLA LIPSCOMB

VAn Roey-Roux, Françoise. 1984. "Enfance de Nathalie Sarraute ou de la fiction à l'autobiographie" in Études Littéraires, 17 (ii), 273-282.

VERCIER, Bruno. 1987. "(Nouveau) Roman et Autobiographie: Enfance de Nathalie Sarraute" in Autobiography in French Literature, French Literature Series, 12, 162-171.

WEI, Keling. 2004. "Pluralité des voix et repentirs autobiographiques: une lecture d'Enfance de Nathalie Sarraute" in Études françaises, 40 (2), 101-114.

Went-Daoust, Yvette. 1987. "Enfance de Nathalie Sarraute ou le pouvoir de la parole" in Les Lettres Romanes, 41, 337-350.

Wiesel, Élie \& François Mittérand. 1995. Mémoire à deux voix. Paris, Éditions Odile Jacob. 
